\title{
Clinical Results of Bone Metastases
}

\author{
Özlem SÖNMEZ
}

Department of Medical Oncology, Acıbadem Mehmet Ali Aydınlar University, Istanbul-Turkey

\section{Introduction}

A bone is the third most common site of metastases after lung and liver, and metastases are often seen in breast, prostate, and lung cancers.[1,2] Many patients with bone metastases experience severe bone pain requiring palliative radiotherapy or pain-killer. Hypercalcemia, nerve compression, pathological fracture, spinal cord compression, and complications requiring orthopedic surgery have negative effects on the quality of life (QoL) of a person. [3] The last four of these complications are defined as skeletal-related events (SRE) and are associated with increased morbidity, higher cost of care, and poor quality of life.[4] The survival rate varies according to tumor type.[5]

Pain is the most common symptom (50\%) of metastatic bone disease (MBD) and includes tumor-induced osteolysis, nerve irritation, production of tumor growth factors and cytokines, and stimulation of ion channels, though its pathophysiological mechanisms remain poorly understood.[6] Bone metastasis affects $28 \%$ of hospitalized patients and $45 \%$ of cancer patients followed at home.[1] The pain develops gradually over weeks or months, intensifies with movement, and occurs more at night, is usually localized to an area, and causes symptoms in the area where metastases are located. For example, while metastases in the skull are associated with cranial nerve palsy and headaches, pelvic and femoral lesions are associated with pain and mechanical imbalance in the lower back and leg area. A study conducted on 1021 patients diagnosed with non-small cell lung cancer in Italy showed that $75 \%$ of patients with bone metastases complained of pain, approximately $50 \%$ of them affected their daily activities and the severity of their pain was proportional to the decrease in quality of life score.[3] Overall, such pain, one of the first symptoms of MBD, is one of the most common types of chronic pain. Considering the severity and duration of the pain, the available treatment methods must be used to control the pain.

Hypercalcemia is the most common metabolic complication of malignant diseases and is clinically important as it may cause morbidity. In most cases, it results in bone destruction, and in $80 \%$ of cases osteolytic metastasis is present. It is observed in $5-10 \%$ of advanced-stage cancer patients, especially those diagnosed with breast cancers, renal adenocarcinomas, squamous cell lung carcinomas, and multiple myelomas. Generally, $80 \%$ of cancer-related hypercalcemia is mediated by parathyroid hormone-related peptide (PTHrP) produced by tumor cells. PTHrP secreted by the tumor cells increases calcium reabsorption in the ascending arm of the loop of Henle and the distal tubule, increases serum calcium levels, and inhibits phosphate reabsorption. [7,8] While the most common symptoms of mild hypercalcemia are nonspecific such as nausea, vomiting, fatigue, constipation, and anorexia, death may result from cognitive disorders, cardiac arrhythmias (e.g., short QT syndrome(SQTS)), and acute renal failure at an advanced stage. [5] It has been reported that approximately half of all patients die within the first month of developing hypercalcemia.[9] Therefore, prompt and appropriate treatments are required. The main goals of treatment are to increase renal calciuresis and prevent pathological bone resorption.

Bone metastases initially cause trabecular disruption and microcracks at the bone surface followed by complete loss of bone integrity. Pathological fractures develop late complication of bone metastases, due to increased bone fragility in $8-30 \%$ of patients. Pathological fractures are reported to develop in $9-29 \%$ of patients with bone metastasis.[10] Though a vertebra
Dr. Özlem SÖNMEZ

Acıbadem Mehmet Ali Aydınlar Üniversitesi,

Tıbbi Onkoloji Anabilim Dalı,

İstanbul-Turkey

E-mail:dr.ozlemus@gmail.com 
is the most common site of bone metastasis, pathological fractures occur most frequently in the ribs and vertebrae, resulting in kyphoscoliosis, height loss, and restrictive lung disease.[5] The 3-year survival rate of patients with bone metastasis and related pathological fractures was found lower than that of patients without pathological fractures.[10]

Spinal cord compression, a common neurological complication of advanced malignancies, is an oncological emergency. Spinal cord compression occurs in 15$20 \%$ of metastatic patients such as prostate, breast, and lung cancer patients, and the median survival of such patients is 3-6 months. In most cases, it occurs very frequently in the dorsal spine as a result of mechanical compression of the spinal cord.[11] Approximately $15 \%$ is seen in the cervical spine, $60 \%$ in the thoracic spine and $25 \%$ in the lumbosacral spine.[12] Symptoms are initially insidious. While symptoms such as back pain, weakness in the lower extremities, and dysesthesia are present initially, over time, they cause progressive neurological deficits such as compression tetraplegia and paraplegia, so early diagnosis and treatment determine the functional outcome. In most cases, corticosteroids, radiotherapy, and surgery are common treatment options.

Clinical experience and few recent studies suggest that the occurrence of bone fractures may be an independent poor prognostic factor for overall survival. The key risk for patients is that metastases occur in weight-bearing areas of the body such as the proximal humerus, cervical spine, and femoral neck. Fractures in these areas reduce the QoL by limiting mobility or causing loss of function.[13]

In conclusion, timely and appropriate management of bone metastasis complications is an important step in optimizing the care and improving the QoL of these patients. In these cases, a multidisciplinary approach including medical oncology, radiation oncology, surgery, orthopedics, and radiology is required.

\section{References}

1. Mercadante S. Malignant bone pain: pathophysiology and treatment. Pain 1997;69(1-2):1-18.

2. Moore RE, Lackman RD. Metastatic Bone Disease. UPOJ 2010;20:117-20.

3. Brodowicz T, O'Byrne K, Manegold C. Bone matters in lung cancer. Ann Oncol 2012;23(9):2215-22.

4. Lipton A, Fizazi K, Stopeck AT, Henry DH, Brown JE, Yardley DA, et al. Superiority of denosumab to zoledronic acid for prevention of skeletal-related events: a combined analysis of 3 pivotal, randomised, phase 3 trials. Eur J Cancer 2012;48(16):3082-92.

5. Coleman RE. Skeletal complications of malignancy. Cancer 1997;80(8 Suppl):1588-94.

6. Coleman RE. Clinical features of metastatic bone disease and risk of skeletal morbidity. Clin Cancer Res 2006;12(20 Pt 2):6243s-9s.

7. Gesek FA, Friedman PA. On the mechanism of parathyroid hormone stimulation of calcium uptake by mouse distal convoluted tubule cells. J Clin Invest 1992;90(3):749-58.

8. Pfister MF, Lederer E, Forgo J, Ziegler U, Lötscher M, Quabius ES, et al. Parathyroid hormone-dependent degradation of type II Na+/Pi cotransporters. J Biol Chem 1997;272(32):20125-30.

9. Ralston SH, Gallacher SJ, Patel U, Campbell J, Boyle IT. Cancer-associated hypercalcemia: morbidity and mortality. Clinical experience in 126 treated patients. Ann Intern Med 1990;112(7):499-504.

10. Böhm P, Huber J. The surgical treatment of bony metastases of the spine and limbs. J Bone Joint Surg Br 2002;84(4):521-9.

11. Robson P. Metastatic spinal cord compression: a rare but important complication of cancer. Clin Med (Lond) 2014;14(5):542-5.

12. Cole JS, Patchell RA. Metastatic epidural spinal cord compression. Lancet Neurol 2008;7(5):459-66.

13. Harvey HA, Cream Lv. Biology of bone metastases: causes and consequences. Clin Breast Cancer 2007;7 Suppl 1:S7-13. 